\title{
Shadowing of gluons in perturbative QCD: A comparison of different models
}

\author{
Jamal Jalilian-Marian ${ }^{1}$ and Xin-Nian Wang ${ }^{2}$ \\ ${ }^{1}$ Physics Department, University of Arizona, Tucson, AZ, USA \\ ${ }^{2}$ Nuclear Science Division, Lawrence Berkeley National Laboratory, Berkeley, CA, USA
}

\begin{abstract}
We investigate the different perturbative QCD-based models for nuclear shadowing of gluons. We show that in the kinematic region appropriate to RHIC experiment, all models give similar estimates for the magnitude of gluon shadowing. At scales relevant to LHC, there is a sizable difference between predictions of the different models.
\end{abstract}




\section{Introduction}

Understanding the initial stages of a ultra-relativistic heavy ion collision is of outmost importance in order to understand the outcome of the proposed heavy ion experiments, soon to go online at RHIC and later to follow at LHC. Understanding the modifications of the parton distributions in nuclei as compared to free nucleons (shadowing) will be an important step towards pinning down the initial conditions of a heavy ion collision.

At high energies ( small $x$ ), there are much more gluons than any other parton species in a hadron/nucleus wavefunction. There are a number of processes which are sensitive to gluon shadowing. High energy production of mini-jets is one such example. Mini-jets will be important at RHIC and will dominate at LHC over soft phenomena. Nuclear shadowing of initial gluon distribution could significantly reduce the initial mini-jet and total transverse energy production. As a result, the subsequent parton thermalization will also be affected due to the reduced initial energy density. Production of heavy quarks is another example where gluon shadowing may make a dramatic difference since the probability for making a heavy quark pair is proportional to the square of gluon distribution function and therefore any depletion in number of gluons will make a significant difference in the number of heavy quark pairs produced.

In recent years, there has been considerable progress made towards understanding gluon shadowing in perturbative QCD. Shadowing of gluons defined as

$$
S\left(x, Q^{2}, b_{t}, A\right) \equiv \frac{x G^{A}\left(x, Q^{2}, b_{t}\right)}{A x G^{N}\left(x, Q^{2}, b_{t}\right)}
$$

can be understood at high energies as a recombination effect due to high gluon number density in the frame where the nucleus is fast, the so called Infinite Momentum Frame (IMF) or as a multiple scattering effect in the rest frame of the nucleus where there is a destructive interference between multiple scattering amplitudes. Off course, so long as one calculates the same physical quantity, with the same approximations made, one must get the same result.

In this note, we continue our numerical study of the shadowing of gluons [四] using two different QCD based formalisms; one is based on an all twist, Wilson renormalization 
group and effective action approach to high gluon density region of QCD as developed in [2, 3, 4]. This approach takes high gluon densities into effect by including and resumming all $n \rightarrow 1$ "hard pomeron" fusion terms in the evolution of both nuclear and nucleon gluon distribution functions. The other formalism is based on a generalization of the MuellerGlauber multiple scattering formalism valid in the rest frame of the nucleus [5, 6, 0, 8] (see also [9]). The two approaches lead to similar but different expressions for the gluon distribution function. The difference between the two approaches is investigated in [10] where it is shown that the effective action and renormalization group approach developed in [2, 3, 4] is more general than the generalized Mueller-Glauber formalism [5, 6, 7, 8, 9] and includes effects which are not present in the latter approach. However, the difference between the two approaches becomes important only at very high energy ( small $x$ ). Here we investigate the predictions of the two approaches for gluon shadowing numerically and show that the difference between the two is negligible in the RHIC kinematic region and becomes more appreciable as one goes to higher energies. For a review of the experimental status of nuclear shadowing, we refer the reader to [11]. For an interesting discussion of the role of coherence in nuclear shadowing and its manifestation in different frames we refer the reader to [12].

This work is organized as follows; in the next section we briefly review the two formalisms followed by a brief recall of our previous results as reported in [何. In section 2, we give the expressions for the nuclear gluon distribution function from the two different formalisms and solve the equations numerically and show our results. We finish with a discussion.

\section{Shadowing in IMF vs. the rest frame}

In the infinite momentum frame, shadowing can be understood as a result of high gluon density at a given impact parameter. The nucleus is highly Lorentz contracted due to its large speed. The small $x$ gluons have large wavelengths compared to the longitudinally Lorentz contracted nucleus size

$$
\lambda \sim \frac{1}{x p^{+}} \gg \frac{2 R}{\gamma}=\frac{2 m R}{p^{+}}
$$


Therefore, small $x$ gluons from different nucleons can spatially overlap and recombine into a higher $x$ gluon. This leads to depletion of the nuclear gluon density as compared to naive expectation that $x G^{A}=A x G^{N}$. In the context of nucleons, this is referred to as saturation of gluon density and slows down the unlimited growth of the gluon distribution function which would otherwise lead to violation of the unitarity bound on physical cross sections [13].

In [4], we derived an evolution equation for the gluon distribution function which takes $n \rightarrow 1$ gluon ladder fusion into account and is the generalization of GLR-MQ model [14] which includes only $2 \rightarrow 1$ ladder recombination. The impact parameter dependent evolution equation is

$$
\frac{\partial^{2}}{\partial y \partial \xi} x G\left(x, Q, b_{\perp}\right)=\frac{3}{\pi^{3}} Q^{2}\left[1-\frac{1}{\kappa} \exp \left(\frac{1}{\kappa}\right) E_{1}\left(\frac{1}{\kappa}\right)\right]
$$

where $\kappa$ is

$$
\kappa=\frac{N_{c} \alpha_{s}}{\pi} \frac{\pi^{3}}{3 Q^{2}} x G\left(x, Q, b_{\perp}\right)
$$

with $y=\log 1 / x$ and $\xi=\log Q^{2}$. The exponential integral function $\mathrm{E}_{1}(x)$ is 15

$$
\mathrm{E}_{1}(x)=\int_{0}^{\infty} d t \frac{e^{-(1+t) x}}{1+t}, \quad x>0 .
$$

In [1], we numerically solved this equation and calculated the nucleon and nuclear gluon distribution function at zero impact parameter. As written, eq. (2) is a generic evolution equation for gluons in either nucleons or nuclei. The distinction between nucleon and nucleus gluon distribution function is made at the initial point $x_{0}$ and $Q_{0}$ after which the nucleon and nucleus gluon distribution function are determined by the evolution equation (see the remarks after eq. 12). We showed in [1] that the non-linearities in the evolution equation induced by the recombination effects are important.

In the rest frame of the nucleus, shadowing is manifested through destructive interference between multiple scattering amplitudes as described by Glauber-Gribov type models. 
This approach was used by Mueller to derive the following expression for the nuclear gluon distribution function in perturbative QCD [5, 6, 7]

$$
\frac{\partial^{2}}{\partial y \partial \xi} x G^{A}\left(x, Q, b_{\perp}\right)=\frac{2}{\pi^{2}} Q^{2}\left[1-e^{-\frac{1}{2} \sigma_{N}^{g g} S\left(b_{t}\right)}\right]
$$

where

$$
\sigma_{N}^{g g} \sim x G_{N}^{D G L A P}\left(x, Q^{2}\right)
$$

is the cross section for scattering of a gluon pair from a nucleon inside the nucleus. It is important to realize that relation (6) holds only at the low gluon density region and will break down once higher twist effects become important.

This corresponds to the following physical picture in the rest frame of the nucleus: a highly virtual DIS probe (or a photon) fluctuates into a gluon pair (or quark anti-quark pair) well before it reaches the nucleus. At small $x$, this pair has a long life time $\tau \sim \frac{1}{m x}$ and coherently scatters off the nucleons as it goes through the nucleus. The destructive interference between the scattering amplitudes reduces the flux of photons as seen by the nucleons sitting inside the nucleus which reduces the nuclear cross sections. The Mueller formula (5) takes into account only the interaction of the fastest (or most energetic) gluon pair with the nucleus. This equation was numerically solved in [6, 7] and we refer the reader there for a comparison. It was shown in [7] that Mueller formula leads to gluon shadowing which is almost independent of the initial non-perturbative shadowing input. It is important to realize that Mueller formula is not a non-linear equation for the gluon distribution function the same way GLR-MQ is. For instance, in Mueller formula, eq. (6) reflects a linear relation between the cross section and the gluon distribution function. This would not hold were there non-linear effects like higher twist terms present. It is also known [6] that Mueller formula over estimates the amount of shadowing. Ayala et al. [6] proposed to include the effects of scatterings of the next-fastest, etc. gluon pairs with the nucleus by iterating the Mueller formula. To do this, one replaces the nucleon gluon distribution function $x G^{N}$ in the exponent of eq. (5) by the nuclear gluon distribution 
function $x G^{A}$. Furthermore, assuming a Gaussian form for the shape function $S\left(b_{t}\right)$, they integrated over the impact parameter to get [6]:

$$
\frac{\partial^{2}}{\partial y \partial \xi} x G^{A}(x, Q)=\frac{3}{4 \pi^{2}} R_{A}^{2} Q^{2}\left[C+\ln \left(\kappa_{a g l}\right)+E_{1}\left(\kappa_{a g l}\right)\right]
$$

where $C \sim 0.57$ is the Euler constant and

$$
\kappa_{a g l}=4 \frac{N_{c} \alpha_{s}}{\pi} \frac{\pi^{3}}{3} \frac{1}{\pi R^{2} Q^{2}} x G^{A}\left(x, Q^{2}\right)
$$

In [6], this replacement is justified by noticing that the new equation has the correct low density properties such that it reproduces DLA DGLAP and GLR. It should be emphasized that this modification of Mueller formula (5) is just an ansatz which seems to produce correct low density limits for the gluon distribution function and, unlike the Mueller formula, was not derived from QCD. It is shown in [8] that, with a specific definition of the gluon distribution function, one can get equation (7) from the $F_{2}$ structure function calculated from the generalized Mueller-Glauber formalism. It is interesting that both approaches predict a slow down in the growth of the gluon distribution function at very small $x$ such that

$$
x G\left(x, Q^{2}\right) \sim \pi R^{2} Q^{2} \ln 1 / x
$$

In the following, in order to compare the two equations, we will use the same Gaussian ansatz as used by [6] to integrate over the impact parameter. We will then solve the two equations numerically starting with the same exact initial conditions and compare the results.

\section{$3 \quad$ Nuclear gluon distribution function}

Here we will use the Gaussian ansatz for the shape function

$$
S\left(b_{t}\right)=\frac{1}{\pi R^{2}} e^{-\frac{b_{t}^{2}}{R^{2}}}
$$

so that $\int d^{2} b_{t} S\left(b_{t}\right)=1$ in order to perform the impact parameter integration in eq. (2). To do so, we first use the relation (4) to rewrite the right hand side of eq. (2) in an 
integral form. We then switch the order of integration and perform the impact parameter integration first and then do the $t$ integration. The result is

$$
\frac{\partial^{2}}{\partial y \partial \xi} x G(x, Q)=\frac{3}{\pi^{3}} \pi R^{2} Q^{2} \exp \left(\frac{1}{\bar{\kappa}}\right) E_{1}\left(\frac{1}{\bar{\kappa}}\right)
$$

where $\bar{\kappa}$ is

$$
\bar{\kappa}=\frac{N_{c} \alpha_{s}}{\pi} \frac{\pi^{3}}{3} \frac{1}{\pi R^{2} Q^{2}} x G(x, Q)
$$

We now solve equations (11) and (7) numerically using the methods described in [1] in full detail. Here we will just briefly highlight the approximations made in [1]. We assumed that at some initial point $x_{0}$ and $Q_{0}$, there is no shadowing. This is motivated by experimental results [11, 16] which show that the ratio $\frac{x G^{A}}{A x G^{N}}=1$ at $x \sim 0.5-0.6$ with weak $Q^{2}$ dependence. We then used the semi-classical approximation to convert our partial differential equations into coupled but ordinary differential equations which can then be solved using Runge-Kutta methods. We refer the reader to [1] for details. The only difference in our choice of parameters in this work is the nuclear radius $R$ which was taken to be $5 \mathrm{fm}$ for $A=200$ in [1] while here we use a more realistic value of $7 \mathrm{fm}$ (more precisely, $R^{A}=R_{0} A^{1 / 3}$ with $R_{0}=1.1 \mathrm{fm}$ being the nucleon radius). To see the effect of integration over the impact parameter on shadowing, we first show our result for shadowing at zero impact parameter in Figure 1.

The difference between the two equations as a function of $x$ and at fixed $Q=5 G e V$ is shown in Figure 2. Comparing Figures 1 and 2, it is clear that averaging over the impact parameter reduces the amount of shadowing as expected. Here, $S^{J K L W}$ refers to the shadowing ratio as defined in (1) as calculated from the solution of eq. (11) while $S^{A G L}$ is calculated from the solution to eq. (]). Also, $S^{G L R}$ is shadowing calculated from the solution of GLR-MQ eq. [14] which is the second term in the expansion of eqs. (11) and (7). The two expressions give very similar results for RHIC $(x \sim 0.01)$ and deviate appreciably only at very small $x$ appropriate to LHC $(x \sim 0.0001)$. For reference, we also show the shadowing ratio calculated from GLR-MQ. As expected, GLR-MQ predicts more shadowing than the other two expressions. 


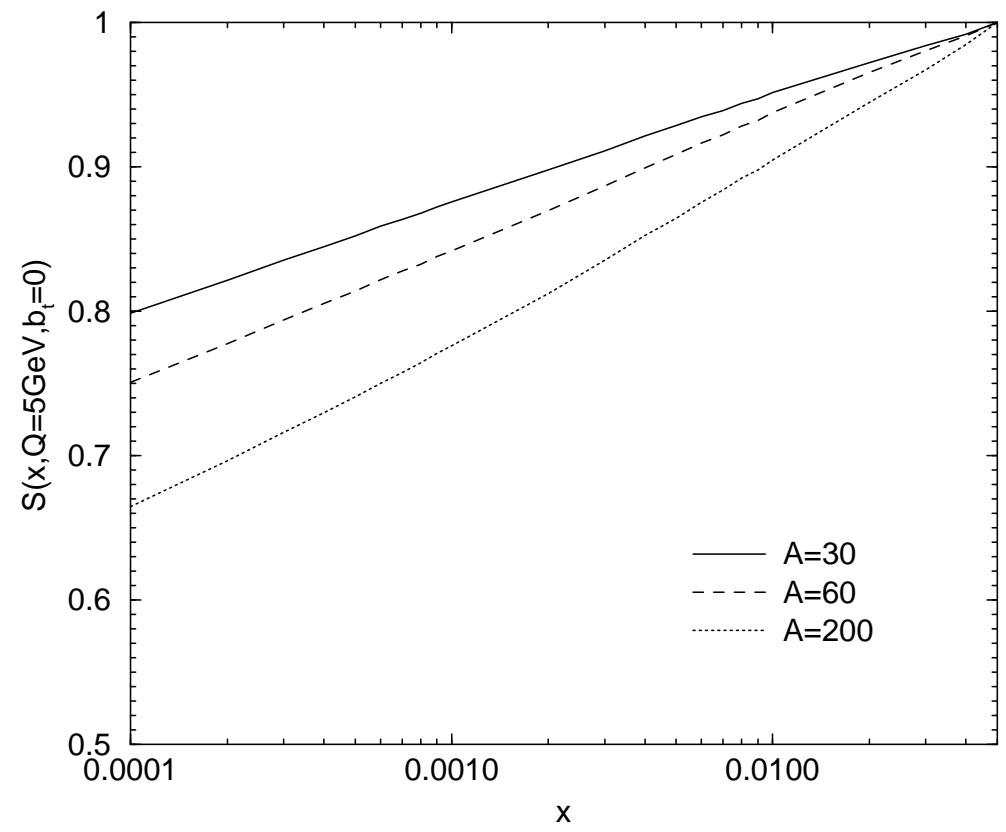

Figure 1: Shadowing from eq. (2) as a function of $x$ at $b_{t}=0$ and $Q=5 \mathrm{GeV}$ for different nuclei.

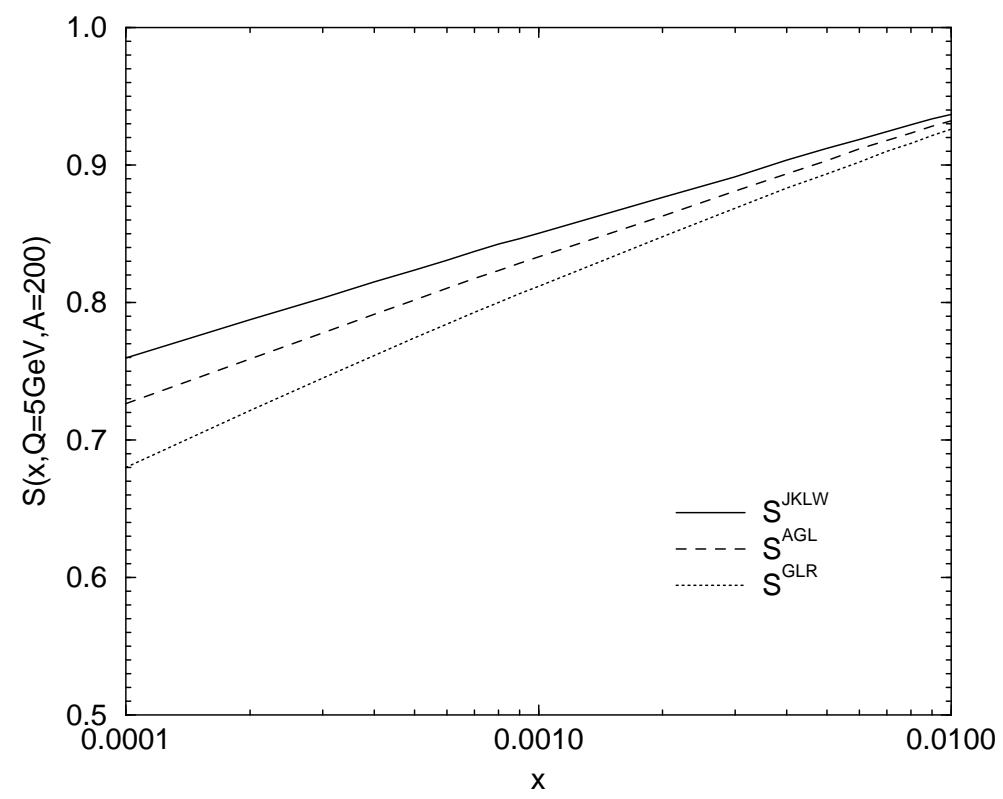

Figure 2: Model dependence of shadowing as a function of $x$ at $Q=5 \mathrm{GeV}$. 
We show the $Q^{2}$ dependence of shadowing at fixed $x$ as predicted by the two equations in Fig. 3. It is interesting to see that both approaches predict very weak $Q^{2}$ dependence of gluon shadowing at RHIC. The difference between the two approaches becomes more pronounced at LHC as $x$ gets smaller. The unphysical trend at low $Q$ is due to both DLA and our semi-classical approximation breaking down at small $Q$ as discussed in full detail in [1].

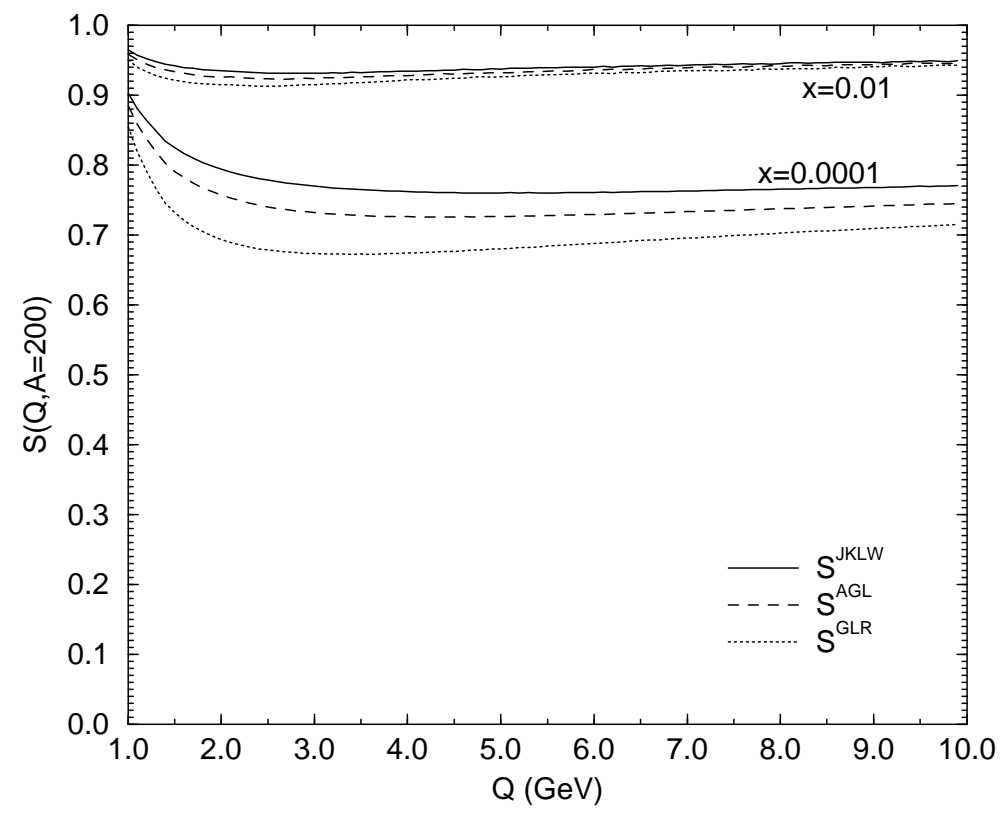

Figure 3: $Q$ dependence of shadowing ratio at RHIC $(x \sim 0.01)$ and LHC $(x \sim 0.0001)$.

The dependence of our results on the choice of the parameterization chosen for the gluon distribution function is shown in Figure 4. In [1], We used the CTEQ [17] parameterization to determine the initial gluon distribution function at scale $x_{0}=0.5$ and $Q_{0} \sim 1 \mathrm{GeV}$. Here we use both CTEQ and GRV94 [18] in order to compare the sensitivity of our results to the choice of parameterization of gluon distribution function available. We chose GRV94 since it is the closest in spirit to DLA approximation employed in all of the perturbative QCD models. Note that GRV94 is known to fail [19] at small values of $x$ and $Q$ but that is not relevant here since we use it at a high value of $x$ where it is known to work. As seen, the two parameterizations predict similar results for RHIC kinematic region while for LHC there is a bigger difference. 


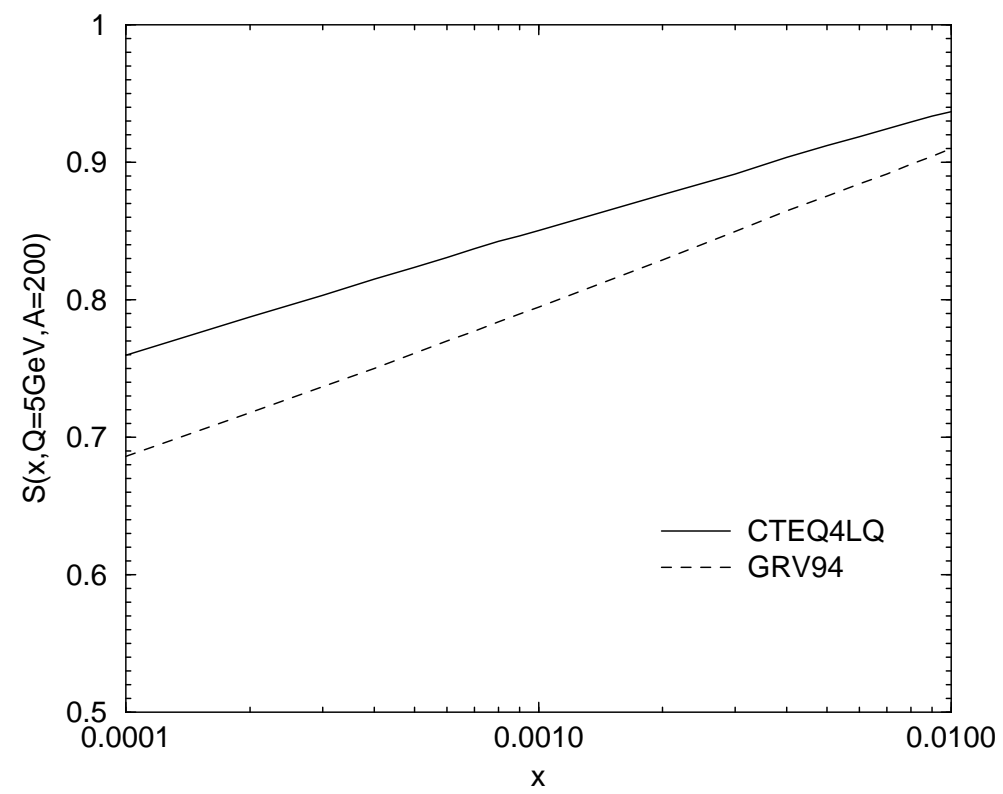

Figure 4: parameterization dependence of shadowing ratio from eq. (11).

\section{Discussion}

We discussed the different perturbative QCD-based models for nuclear gluon distribution function and numerically investigated their predictions for $x$ and $Q$ dependence of shadowing in the kinematic region appropriate to RHIC and LHC. We showed that predictions of different models for shadowing of gluons at RHIC are comparable while the difference at LHC can be of order $\sim 10 \%$ for Gold or Lead. An important point which needs to be investigated further is inclusion of initial shadowing of gluons due to non-perturbative effects at the starting point of evolution $x_{0}$ after which the perturbative evolution takes place. This is currently under investigation and will be reported on later [20]. The more experimentally relevant quantity to investigate is the shadowing of the nuclear structure function $F_{2}^{A}$ since shadowing of gluons is not directly observable. The all twist $F_{2}^{N}$ and $F_{2}^{A}$ as well as the longitudinal structure function $F_{L}$ were computed in [2] at the classical level. Including the quantum loop effects due to gluons is straight forward and is currently under investigation [21]. One can then predict the experimentally measured shadowing ratio $F_{2}^{A} / F_{N}^{2}$ for different nuclei at different $x, Q^{2}$ as well as the longitudinal structure functions. 


\section{Acknowledgments}

We would like to thank I. Sarcevic for discussions. J. J-M. was supported by U.S. Department of Energy under contract No. DE-FG03-93ER40792. X-N. W. was supported by the Director, Office of Energy Research, Office of High Energy and Nuclear Physics Division of the Department of Energy, under contract No. DE-AC03-76SF00098 and DEFG02-87ER40328.

\section{References}

[1] J. Jalilian-Marian and X-N. Wang, Phys. Rev. D60, 054016 (1999).

[2] L. McLerran and R. Venugopalan, Phys. Rev. D49, 335 (1994); D49, 2233 (1994), D59, 094002 (1999), R. Venugopalan, Acta Phys.Polon. B30, 3731 (1999).

[3] A. Ayala, J. Jalilian-Marian, L. McLerran and R. Venugopalan, Phys. Rev. D52, 2935 (1995); D53, 458 (1996).

[4] J. Jalilian-Marian, A. Kovner and H. Weigert, Phys. Rev. D59, 014015 (1999); J. JalilianMarian, A. Kovner, A. Leonidov and H. Weigert, Phys. Rev. D59, 014014 (1999), 034007 (1999), Nucl. Phys. B504, 415 (1997); J. Jalilian-Marian, A. Kovner, L. McLerran and H. Weigert, Phys. Rev. D55, 5414 (1997).

[5] A.H. Mueller, Nucl. Phys. B335, 115 (1990).

[6] A.L. Ayala, M. B. Gay Ducati and E. M. Levin, Nucl. Phys. B493, 305 (1997); B511, 355 (1998).

[7] Z. Huang, H.J. Lu and I. Sarcevic, Nucl. Phys. A637, 70 (1998).

[8] Y. Kovchegov, Phys. Rev. D54, 5463 (1996); D55, 5445 (1996); D60, 034008 (1999).

[9] I. Balitskii, Nucl. Phys. B463, 99 (1996).

[10] A. Kovner, J. G. Milhano and H. Weigert, hep-ph/0004014.

[11] M. Arneodo, Phys. Rep. 240, 301 (1994).

[12] G. Piller and W. Weise, Phys. Rep. 330, 1 (2000).

[13] M. Froissart, Phys. Rev. 123, 1053 (1961).

[14] L.V. Gribov, E.M. Levin and M.G. Ryskin, Phys. Rep. 100, 1 (1983); A.H. Mueller and J.W. Qiu, Nucl. Phys. B268, 427 (1986); J.W. Qiu, Nucl. Phys. B291, 746 (1987).

[15] M. Abramowitz and I. Stegun, "Handbook of Mathematical Functions", Dover, New York, 1972.

[16] M.R. Adams, et.al., Phys. Rev. Lett. 68, 3266 (1992).

[17] H.L. Lai. et al., Phys. Rev. D55, 1280 (1997).

[18] M. Gluck, E. Reya and A. Vogt, Z. Phys. C67, 433 (1995).

[19] A. Caldwell, DESY Theory Workshop, DESY, October 1997.

[20] J. Jalilian-Marian and I. Sarcevic, in preparation.

[21] J. Jalilian-Marian and R. Venugopalan, in preparation. 